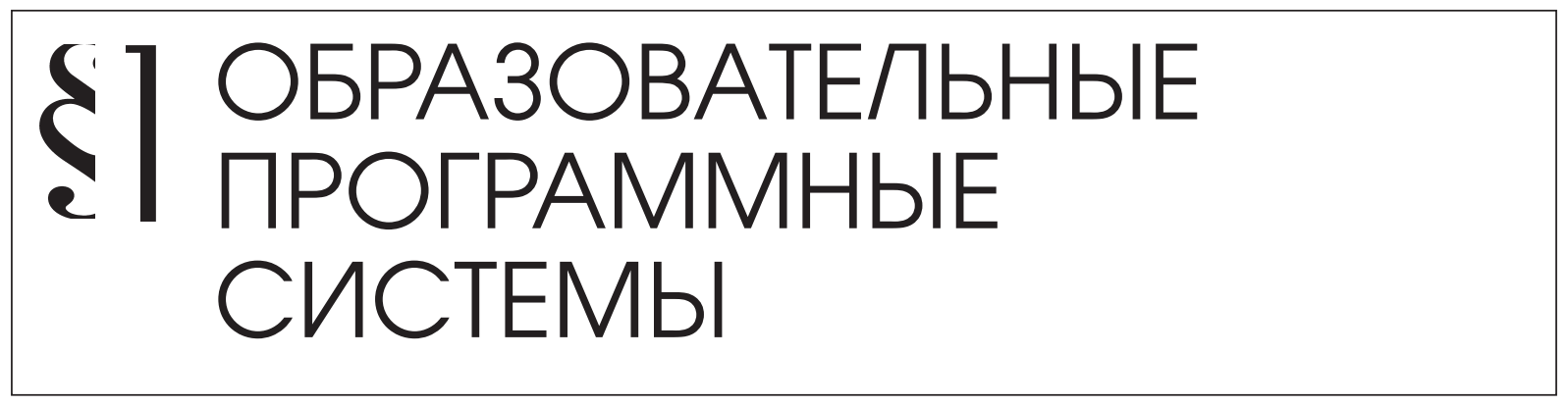

Гуляихина E.C.

\title{
РАЗРАБОТКА ВИРТУАЛЬНОГО ПРИБОРА ДЛЯ КОМПЬЮТЕРНОЙ СИМУЛЯЦИИ В СФЕРЕ КЛИНИЧЕСКОЙ ЛАБОРАТОРНОЙ ДИАГНОСТИКИ
}

Аннотация: Предметом исследования является процесс создания компьютерной симуляции, имитирующей проведение клинического лабораторного анализа биологических жидкостей с помощью биохимического анализатора Stat Fax ${ }^{\circledR}$ 1904+. Основное внимание уделяется ключевым компонентам обучающего симулятора и алгоритму работы с ним. Представлены особенности разработки виртуального прибора, обусловленные его обучающим назначением, и заключающиеся в разном уровне подготовки пользователей системы и возможностью некорректного ввода данных. Автор предлагает создать три вида пользователей и разработать функции программы, обеспечивающие достоверность данных и соблюдение алгоритма работы с виртуальным прибором. Изложены этапы создания компьютерного симулятора, основными из которых являются моделирование системы, трансляция модели и анализ результатов разработки. Onисаны методы определения концентрации вещества в исследуемом образце, симулируемые компьютерной программой, и приведены соответствующие формулы для вычислений. Представлена блок-схема, отражающая прочесс работы компьютерной программы, на основе которой реализован геометрический дизайн форм приложения и функции системы. Созданный компьютерный симулятор может применяться в образовательном процессе на этапе формирования практических навыков обучающихся, а также при повышении уровня профессионализма работников клинических лабораторий. Результаты проведенного исследования могут быть использованы при создании обучающих симуляторов не только для проведения биохимического анализа, но и для других областей профессиональной деятельности, требующих применения навыков работы с высокотехнологичным оборудованием. Ключевые слова: компьютерная симуляция, обучающий симулятор, биохимический анализатор, Stat Fax ${ }^{\circledR}$ 1904+, Visual Basic, лабораторная диагностика, моделирование, трансляция модели, Microsoft Visual Studio, объектная модель

Abstract: The subject of research is the process of creating a computer simulation simulating the conducting a clinical laboratory analysis of biological fluids using a biochemical analyzer Stat 
Fax ${ }^{\circledast} 1904+$. Particular attention is driven to the components of the training simulator and to the algorithm for work with it. The article presents features of developing the virtual instrument, caused by its teaching assignment, which consist of the different training levels of users of the system and the possibility of incorrect input data. The author proposes to create three types of users and design features of the software to ensure the accuracy of the data and compliance with the algorithm of the virtual appliance. The paper shows stages of making a computer simulator, such as modeling the system, translation of the model and analyzing the results of the development. The author describes concentrations of a substance in a sample simulated by the software and gives the corresponding formulas for calculations. The study presents a flowchart showing the process of operation of the computer program. The geometric design of application forms and functions of the system is based on this flowchart. A computer simulator created can be used in education at the stage of forming practical skills of students as well as raising the level of professionalism of clinical laboratories. The results of the study can be used in creating training simulators not only for biochemical analysis, but also for other areas of professional activities requiring the use of skills in working with high-tech equipment.

Keywords: model translation, modelling, laboratory diagnostics, Visual Basic, Stat Fax ${ }^{\circledR} 1904+$, biochemical analyzer, training simulator, computer simulation, Microsoft Visual Studio, object model

Исследование проводилось в рамках программы «Участник молодежного научно-инновационного конкурса», которую организует федеральное государственное бюджетное учреждение «Фонд содействия развитию малых форм предприятий в научно-технической сфере», при финансовой поддержке проекта «Эмуляция процесса измерения количественных и качественных характеристик биологических жидкостей колориметрическим методом с использованием программы-эмулятора» по договору №5622ГУ2/2014.

\section{Введение}

Имитация алгоритма и особенностей работы с профессиональным оборудованием является основой для создания компьютерных симуляторов, позволяющих воспроизвести полный цикл работы с прибором, начиная с его включения и заканчивая анализом произведенных действий. В последнее время обучающие симуляторы становятся все более популярными и востребованными, поскольку позволяют сделать более эффективным процесс для подготовки специалистов в различных областях профессиональной деятельности [1; 3; 4]. Образовательные учреждения не являются исключением и применяют их для повышения уровня сформированности практических навыков будущих специалистов. Среди востребованных областей применения компьютерных симуляторов являются профессии, предполагающие работу с высокотехнологичным оборудованием и с дорогостоящим оснащением профессиональной деятельности. К этой сфере относятся 
медицинские специалисты, профессиональное образование которых отличается спецификой формирования практических навыков и необходимостью работы с биологическими образцами. Организация такого процесса может быть упрощена посредством компьютерных симуляторов, имитирующих количественные и качественные характеристики используемых образцов. Например, ученые Мадридского университета Комплутенсе разработали веб-симулятор для исследования образцов крови лабораторных крыс в рамках курса физиологии [5]. Внедрение этой технологии позволило минимизировать финансовые затраты, соблюсти биоэтические принципы (сохранение жизни лабораторных животных), а также отработать практические навыки студентов до проведения экспериментов. Однако в сфере отечественного медицинского образования наблюдается явный дефицит виртуальных приборов, и это несмотря на большое разнообразие и высокую стоимость аппаратного обеспечения в данной отрасли, требующей высокий уровень профессионализма персонала [7]. Среди мало разработанных направлений находится создание обучающих компьютерных симуляторов для проведения анализа биологических жидкостей с использованием биохимического анализатора.

Многие российские медицинские вузы и лаборатории клинической диагностики используют биохимические анализаторы серии Stat Fax ${ }^{\circledR}$ американской компании «Awareness Technology Inc.», которые являются фотометрами под управлением микропроцессора. В проведенном исследовании в качестве образца был взят прибор Stat Fax ${ }^{\circledR}$ 1904+ для создания компьютерного симулятора, полностью имитирующего деятельность по проведению биохимического анализа. Данный биохимический анализатор используется медицинскими учреждениями, которые являются базовыми для прохождения практики студентами Волгоградского государственного медицинского университета, не имеющими достаточных возможностей для отработки навыков работы с прибором.

\section{Принцип работы объекта симуляции}

Биохимический анализатор Stat Fax ${ }^{\circledR}$ 1904+ (рис. 1) предназначен для измерения концентрации веществ (например, холестерин, билирубин и т.д.) в биологических жидкостях. Достаточными условиями для проведения измерений являются: наличие набора реагентов для каждого вещества, собственно прибора и исследуемого образца с жидкостью. К набору реагентов прилагается инструкция с описанием составляющих набора и метода, которым следует проводить измерение. После подготовки пробирок, необходимых для измерения, лаборант загружает их в определенном порядке в термостатирующую ячейку прибора. Взаимодействие пользователя с биохимическим анализатором происходит с помощью дисплея и клавиатуры. Результаты измерений доступны на бумажной ленте печатного устройства биохимического анализатора. 


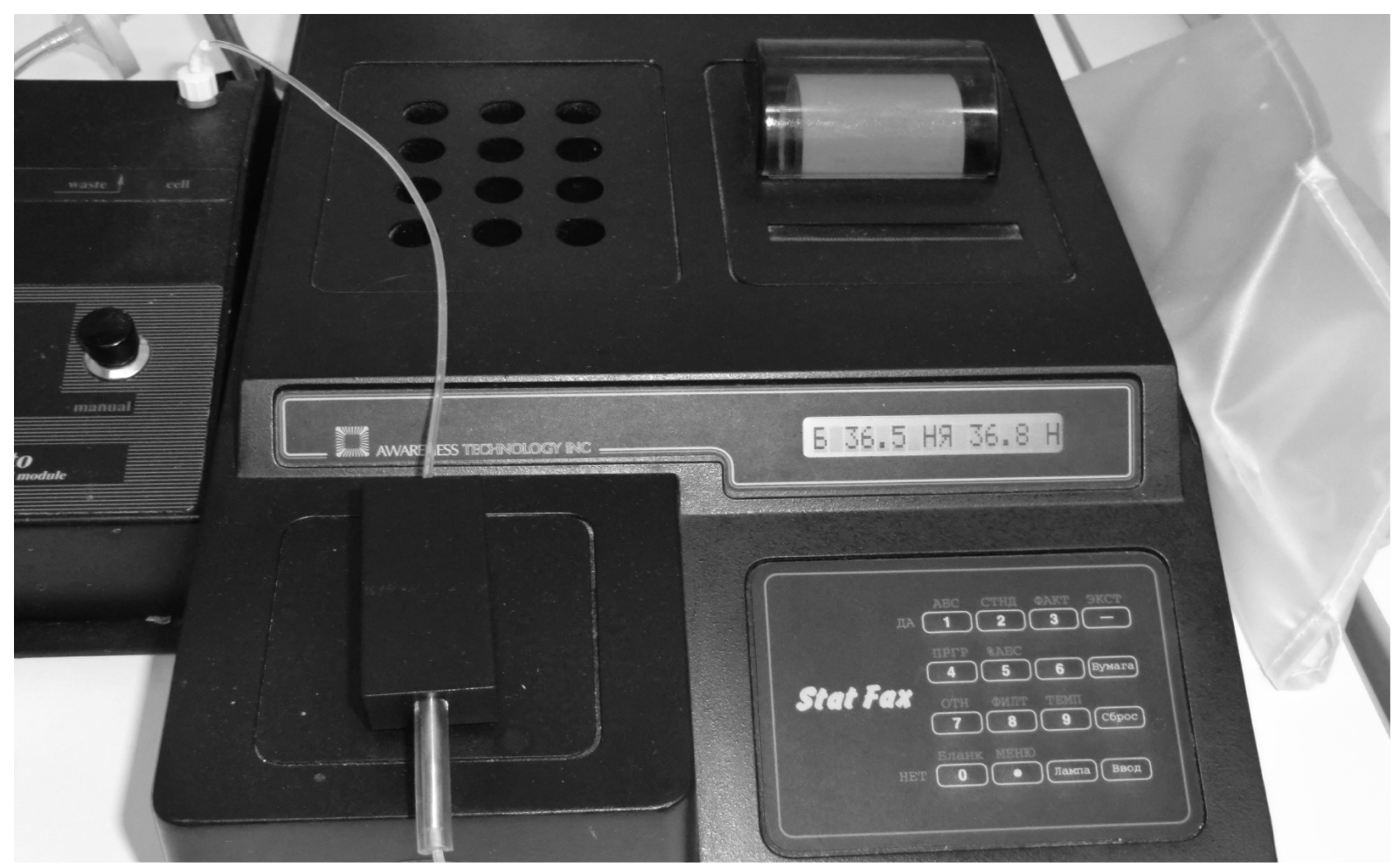

Рис. 1. Внешний вид биохимического анализатора Stat Fax ${ }^{\circledR} 1904+$.

Биохимический анализатор дает возможность измерить концентрацию вещества в биологической жидкости на основе изменения интенсивности окраски (оптической плотности) химического соединения. Измерение оптической плотности (абсорбции) данным прибором основано на способности поглощения химическим соединением лучистой энергии определенных длин волн. После прохождения светового потока, фокусируемого линзой, через исследуемую пробу, фотодетектор преобразует энергию проходящего света в электрические сигналы, которые затем усиливаются и интерпретируются. Далее программа умножает полученное значение абсорбции на фактор перерасчета и выводит значение концентрации вещества. Фактор может быть получен одним из способов: ввод значения с клавиатуры или измерение стандарта (смесь, в которой концентрация исследуемого вещества известна с высокой точностью). Второй способ предполагает вычисление отношения концентрации стандарта к его абсорбции, измеренной прибором. Когда фактор перерасчета становится известным для программы, можно переходить к измерению концентрации вещества в исследуемом образце. Этот процесс осуществляется методом измерения по конечной точке (endpoint colorimetric assay), предполагающее замер в момент, когда реакция прекращается, и значение оптической плотности становится вполне постоянной величиной, или кинетическим методом (kinetic colorimetric assay). Для программы в первом случае это означает, что вычисление происходит сразу после измерения абсорбции образца путем умножения ее на фактор, а во втором - сначала необходимо определить промежутки времени для измерения абсорбции образца. Следует 
отметить, что фотометрической метод биохимического анализа может сопровождаться систематической ошибкой, оказывающей влияние на конечный результат реактива [6]. Для ее исключения в биохимическом анализаторе предусмотрено измерение относительно бланка (смесь, в которой концентрация исследуемого вещества равна 0). Прибор сообщает о моменте измерения бланка через дисплей, и при расчете концентрации исследуемого вещества автоматически вычитает значение абсорбции бланка из значения оптической плотности конечной точки исследуемого образца. Расчетные формулы методов вычисления концентрации вещества представлены в таблице 1 , где $C_{s t}$ и $D_{s t}$ - концентрация и абсорбция стандарта соответственно, $C_{i}$ - концентрация $i$-того образца, $F$ - фактор, $D_{i}$ - абсорбция образца, $D_{i_{2}}$ - абсорбция образца в начале заданного отрезка времени; $D_{i_{2}}$ - абсорбция образца в конце заданного отрезка времени.

Таблица 1.

\begin{tabular}{|c|c|c|c|c|c|}
\hline \multirow[b]{2}{*}{$\begin{array}{c}\text { Статус } \\
\text { фактора }\end{array}$} & \multirow[b]{2}{*}{$\begin{array}{c}\text { Название } \\
\text { метода }\end{array}$} & \multirow[b]{2}{*}{$\begin{array}{c}\text { Вид } \\
\text { измерения }\end{array}$} & \multicolumn{2}{|c|}{$\begin{array}{c}\text { Промежуточные } \\
\text { вычисления }\end{array}$} & \multirow{2}{*}{$\begin{array}{c}\text { Формула } \\
\text { для расчета } \\
\text { концентрации }\end{array}$} \\
\hline & & & $\begin{array}{c}\text { Расчет } \\
\text { фактора }\end{array}$ & $\begin{array}{c}\text { Определение } \\
\text { абсорбции } \\
\text { вещества }\end{array}$ & \\
\hline \multirow[b]{2}{*}{ Неизвестен } & \multirow{2}{*}{$\begin{array}{c}\text { По } \\
\text { стандарту }\end{array}$} & $\begin{array}{l}\text { По конечной } \\
\text { точке }\end{array}$ & \multirow{2}{*}{$\frac{C_{s t}}{D_{s t}}$} & \multirow{4}{*}{$D_{i}=D_{e p}-D_{b}$} & $C_{i}=F^{*} D_{i}$ \\
\hline & & $\begin{array}{c}\text { Кинетический } \\
\text { метод }\end{array}$ & & & $C_{i}=\frac{D_{i_{1}}-D_{i_{2}}}{\min } * F$ \\
\hline \multirow[b]{2}{*}{ Известен } & \multirow{2}{*}{$\begin{array}{c}\text { По } \\
\text { фактору }\end{array}$} & $\begin{array}{c}\text { По конечной } \\
\text { точке }\end{array}$ & - & & $C_{i}=F^{*} D_{i}$ \\
\hline & & $\begin{array}{c}\text { Кинетический } \\
\text { метод }\end{array}$ & - & & $C_{i}=\frac{D_{i_{1}}-D_{i_{2}}}{\min } * F$ \\
\hline
\end{tabular}

\section{Моделирование компьютерной системы}

Одними из основных этапов создания компьютерного симулятора являются сбор исходных данных, позволяющих создать модель системы, и последующая трансляция полученной модели [2]. Общий алгоритм программы и технология работы с симулятором были определены после беседы с лаборантом, непосредственного наблюдения процесса измерения концентрации вещества с помощью прибора Stat Fax ${ }^{\circledR}$ 1904+ и изучения инструкции пользователя прибора. В процессе сеанса работы пользователь системы выбирает пробирки согласно запросам прибора, отображаемых на его дисплее, настраивает прибор, задает параметры теста и получает результаты измерений. Поскольку компьютерный симулятор имеет обучающее назначение и предназначен для работы пользователей различного уровня подготовки, то целесообразно предусмотреть два типа пользователей системы для работы с симулятором прибора - стажер и лаборант - и 
один тип пользователя системы - администратор (рис. 2).

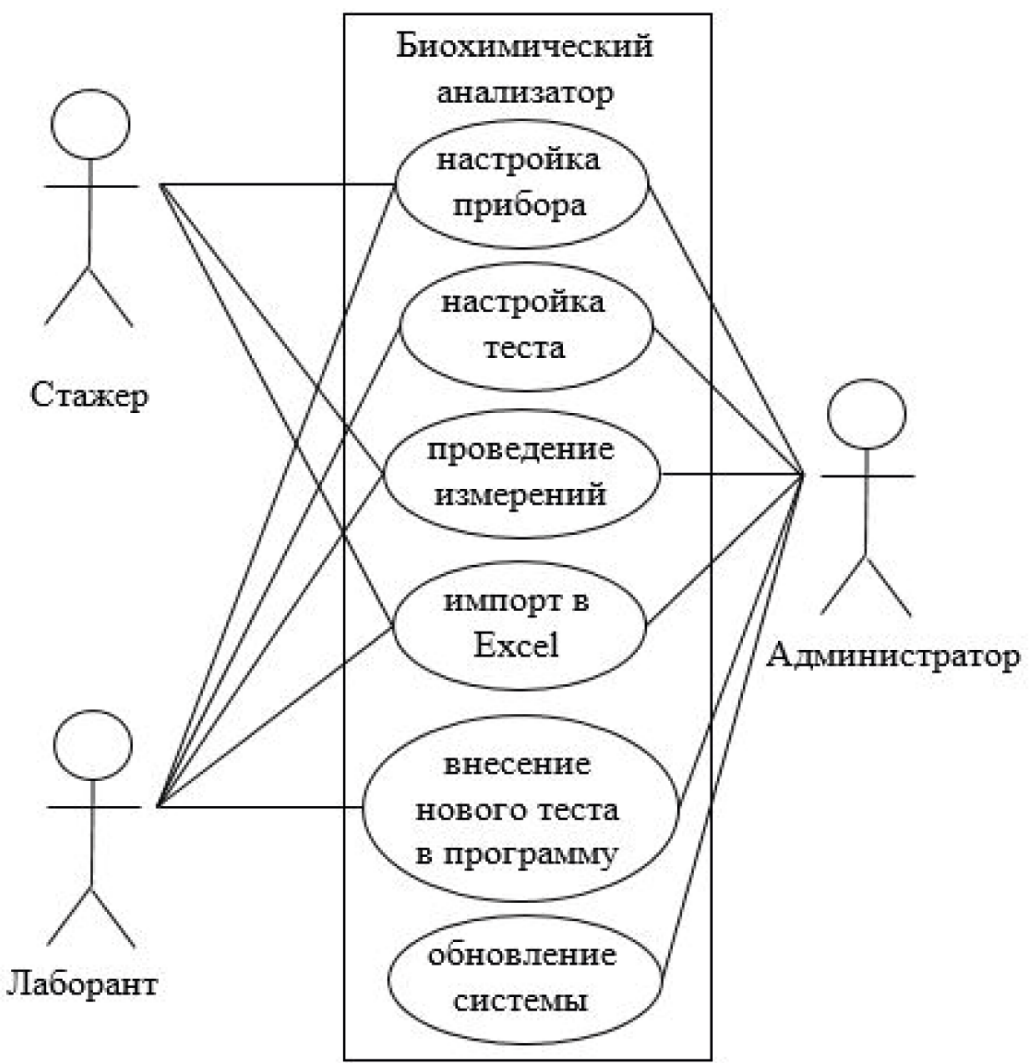

Рисунок 2. Диаграмма прецедентов модели симулятора биохимического анализатора.

При знакомстве с виртуальным прибором и проведении первых измерений с ним пользователь еще не знаком с технологией настройки тестов, поэтому его действия следует ограничить только проведением заранее сохраненного теста, обучением настройке прибора и получением результатов. Более опытный пользователь может определять параметры теста на основе методик, прилагаемых к набору реагентов. Виртуальный прибор содержит названия двадцати тестов, которые могут быть настроены. Однако при необходимости администратор системы может произвести не только обновление системы, но и добавление параметров нового теста. При создании компьютерной программы для каждого типа пользователя должен быть создан отдельный модуль.

Технология работы с виртуальным прибором строится по определенному принципу, который предполагает вариации в зависимости от выбранного метода измерения - кинетический метод или метод по конечной точке, измерение только абсорбции, с использованием известного фактора или стандарта. В процессе настройки параметров теста прибор также выдает запрос о введении дополнительных параметров: границ норм и линейности, выборе единиц измерения. Структура этого процесса представлена на рисунке 3. 


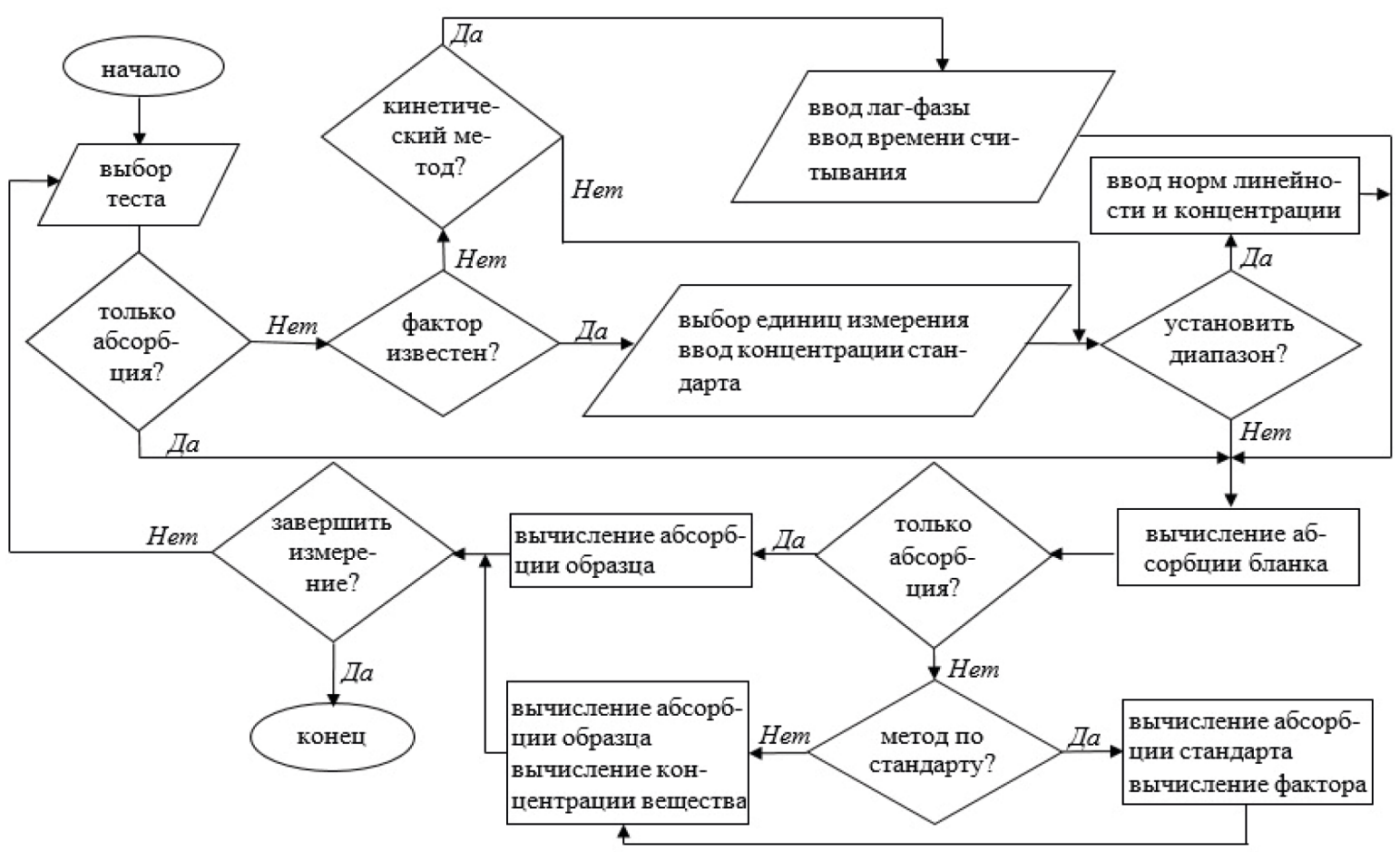

Рис. 3. Блок-схема компьютерного симулятора.

Особый интерес представляют входные и выходные данные системы. Первый вид данных состоит из значений качественных характеристик исследуемых веществ, позволяющих проводить вычисления на основе формул таблицы 1. Ключевым значением для этих формул выступает абсорбция исследуемого образца, являющаяся уникальным для каждого теста и измеряемая на определенной длине волны в зависимости от выбранного фильтра. Значение абсорбции может быть задано генератором случайных чисел из числового промежутка, рассчитанного с помощью электронной таблицы на основе известных границ нормы и абсорбции бланка. Анализ более двухсот результатов различных тестов, осуществленных на данном приборе, позволил собрать возможные значения абсорбции бланка и стандарта и вычислить значение фактора, промежутки абсорбции образца на основе формул таблицы 1. Проведенные вычисления легли в основу таблицы, содержащей в последнем столбце числовые значения для компьютерного симулятора, фрагмент которой изображен на рисунке 4. Результаты промежуточных вычислений хранятся в переменных программы.

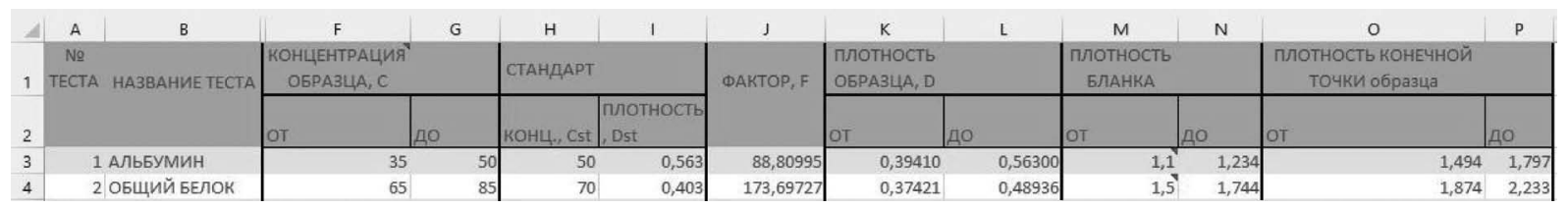

Рис. 4. Фрагмент таблицы с числовыми значениями для генератора случайных чисел. 
Следует отметить, то сведения о настройках прибора и заданных параметрах теста компьютерная программа не хранит согласно обучающему назначению симулятора. Однако, прибор Stat Fax ${ }^{\circledR}$ 1904+ обладает энергонезависимой памятью и способен запоминать эти сведения, поэтому необходимо симулировать это свойство хотя бы в незначительной степени. Реализация такой симуляции возможна с помощью электронной таблицы, которая будет хранить настройки теста на персональном компьютере или в течение сеанса работы с ним или на жестком диске. В случае работы с компьютерным симулятором пользователя «Лаборант» сведения загружаются из соответствующего модуля программы, а работа в режиме «Стажер» предполагает загрузку файла электронной таблицы с необходимыми данными.

Выходными данными компьютерной системы являются результаты теста, выводимые в текстовую область (симуляция печатного устройства), которые должны быть доступны для их дальнейшей обработки. Поэтому была предусмотрена выгрузка данных в электронную таблицу, позволяющую проводить их анализ. Таким образом, для работы компьютерного симулятора потребовалось два файла электронной таблицы: один - для хранения параметров теста, второй - для выгрузки данных.

\section{Трансляция модели симулятора}

\section{Геометрический дизайн}

Инструментальным средством разработки компьютерного симулятора проведения биохимического анализа с помощью прибора Stat Fax ${ }^{\circledR}$ 1904+ выступила среда Microsoft Visual Studio Express 2013, а языком программирования - Visual Basic. Данный симулятор является Windows-приложением и состоит из нескольких форм: стартовая форма, форма для выбора пользователя системы, основная форма с виртуальным прибором, форма для работы администратора системы. В основной форме была создана рабочая поверхность стола, которая содержит изображения прибора с действующей клавиатурой и дисплеем, устройством вывода результатов измерения на бумагу, пробирок с соответствующими им кнопками: бланк, стандарт и образец, кнопки питания и выгрузки в MS Excel ${ }^{\circledR}$.

Элементы интерфейса, включая кнопки, раскрывающиеся списки, изображения и другие элементы, выступили в качестве компонентов системы, оперирующими глобальными переменными. Каждый элемент имеет свои настройки по умолчанию, а некоторые из них - локальные переменные. Создание иерархической структуры системы и логических взаимоотношений между компонентами было осуществлено с помощью объектной структуры, изображенной в правой части окна программы (рис. 5). Такая организация рабочей области позволила своевременно определить свойства компонентов. 


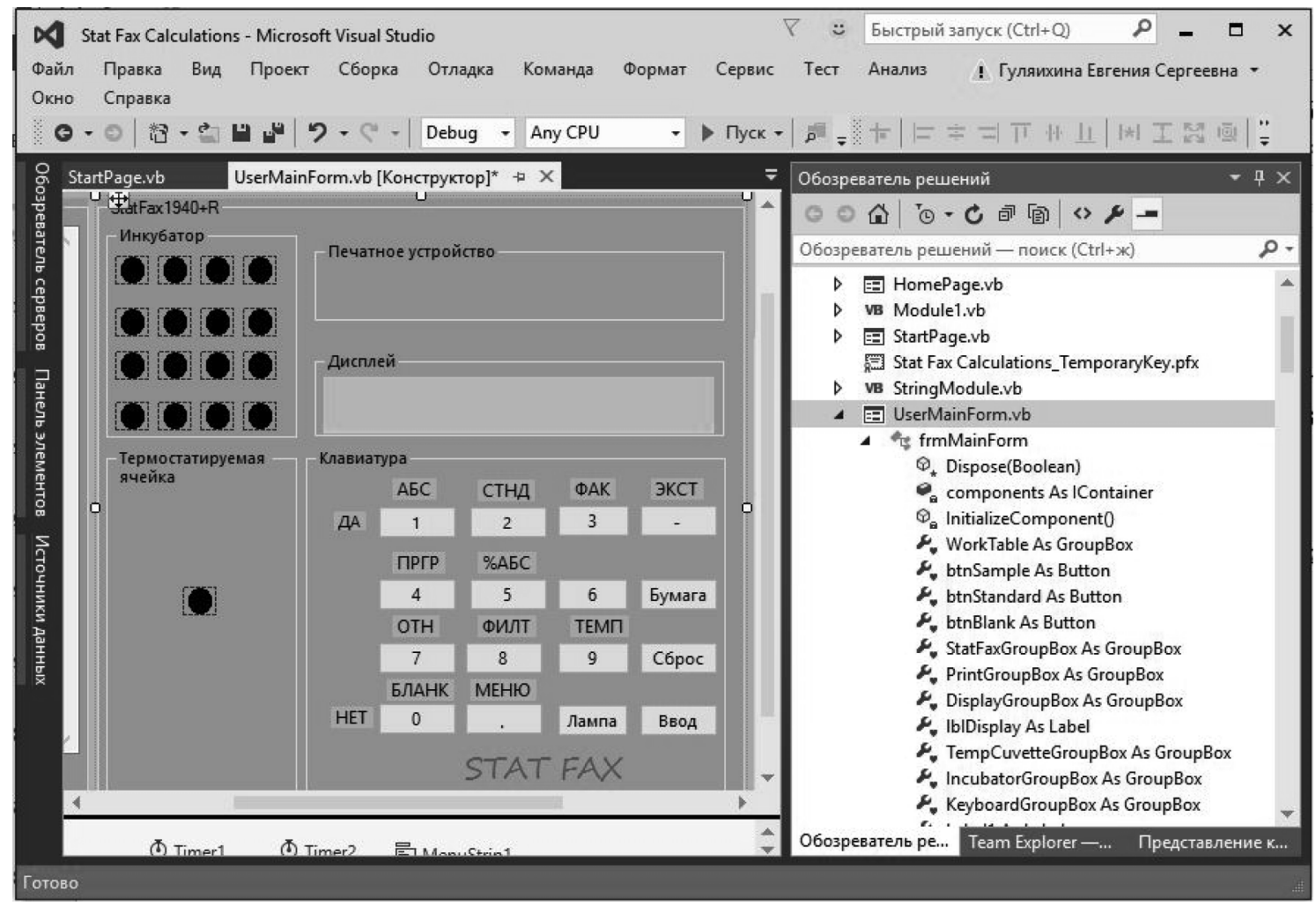

Рис. 5. Геометрический дизайн главного окна.

\section{Реализация функций системы}

Основополагающим классом данной компьютерной программы является класс «TestData», определенный на VB.NET с помощью элемента «Структура» и предназначенный для данных какого-либо теста (листинг 1). Для определения таких атрибутов экземпляров этого класса, как название теста, метод вычисления, единицы измерения и название параметра теста, использованы массивы.

\section{Public Structure TestData ' структура содержит все настройки теста}

Public intFilter As Integer ' хранит значение фильтра

Public strSelectedTest As String ' хранит название выбранного теста

Public strSelectedMethod As String ' хранит название выбранного метода

Public blnSampleBlankMode As Boolean ' истинно при режиме бланка по пробе

Public strSelectedUnitCode As String ' хранит код единиц измерения

Public dblKeyLowNormal As Double ' хранит нижнюю грань нормы

Public dblKeyHighNormal As Double ' хранит верхнюю грань нормы

Public dblKeyLoLinearity As Double ' хранит нижнюю грань линейности

Public dblKeyHILinearity As Double ' хранит верхнюю грань линейности

Public intLagTime As Integer ' хранит время лаг-фазы

Public intReadTime As Integer ' хранит время для считывания 
Public dblStandardConcentration As Double ' хранит значение концентрации стандарта

Public dblFactorValue As Double ' хранит значение фактора

Public blnsavedinfile As Boolean ' истинно в случае сохраненного теста

Листинг 1. Определение структуры данных.

После задания глобальных переменных была решена одна из задач, требующих больших затрат времени - программирование клавиш виртуального прибора. Для этого был определен алгоритм для каждой клавиши и на его основе создан код программы. Цифровые клавиши различаются по количеству функций, которые они выполняют. Например, клавиша «1», кроме задания числовых значений, метода расчета и длины волны, определяет значение «ИСТИНА» для булевых переменных. Если вопрос предполагает ответ в формате «Да/Нет», то при нажатии клавиши «1» на дисплее появляется надпись: «ДА» (кроме случаев при выборе метода и задании числовых значений), и соответствующая переменная принимает значение «ИСТИНА». В свою очередь, клавиша «8» помимо задания числовых значений выводит на печать (в текстовую область) список фильтров и соответствующих им клавиш. Среди текстовых клавиш наибольшей значимостью обладает клавиша «Ввод», поскольку она реализует наибольшую часть алгоритма программы, связанную с процессом настройки параметров теста. Также особой функциональностью обладает клавиша «ЭКСТ». С ее помощью пользователь может сохранить, редактировать или удалить тест, а также ввести дату/время, выключить принтер и т.д.

Симуляция собственно процесса измерения концентрации вещества была осуществлена с помощью таймеров и функций. Например, при нажатии на кнопку «Проба» на дисплее виртуального прибора выводится надпись: «Считывание...», которая задерживается с помощью таймера на несколько секунд. В это же время происходит вычисление концентрации искомого вещества по формулам таблицы 1 и данным таблицы расчета оптической плотности образца (листинг 2).

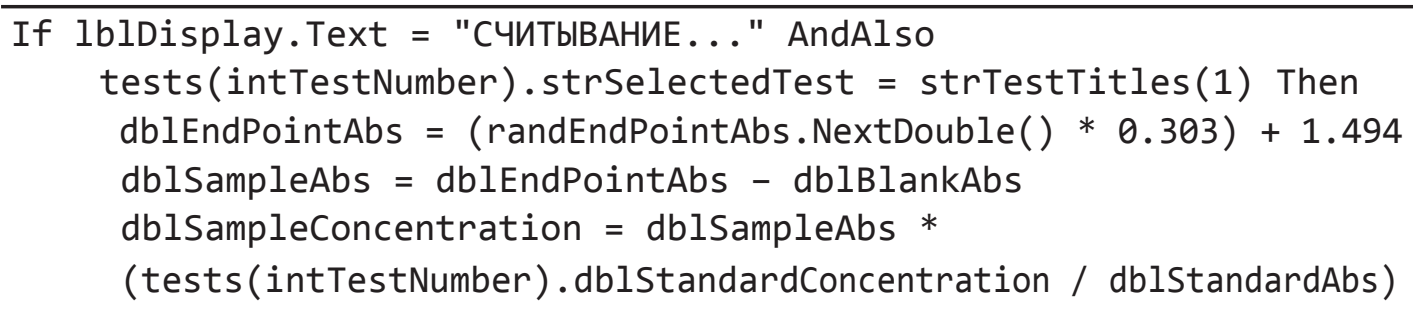

Листинг 2. Вычисление концентрации вещества.

Для загрузки данных из сохраненных параметров теста была создана процедура, открывающая файл программы MS Excel ${ }^{\circledR}$ и присваивающая значения определенным переменным. Использование этой программы для симуляции памяти прибора и выгрузки результатов тестов реализовано с помощью объектной модели. 
Public xlApp As Microsoft.Office.Interop.Excel.Application

Public xlBook As Microsoft.Office.Interop.Excel.Workbook

Public xlExportBook As Microsoft.Office.Interop. Excel.Workbook

Public xlSheet As Microsoft.Office.Interop.Excel.Worksheet

Public xlExportSheet As Microsoft.Office.Interop.Excel.Worksheet

Public xlSavedTestBook As Microsoft.Office.Interop.Excel.Workbook

Public xlSavedTestSheet As Microsoft.Office.Interop.Excel.Worksheet

Public strSaveFilePath As String

Листинг 3. Использование объектной модели MS Excel ${ }^{\circledR}$.

\section{Результаты разработки}

Основная часть тестирования компьютерного симулятора проводилась посредством реализации различных сценариев и проверки функциональности виртуального прибора. В результате было выявлено, что компьютерный симулятор реализует основные методы измерений концентрации вещества, запрограммированные в приборе, и отражает алгоритм проведения биохимического анализа. После включения виртуального прибора пользователь может изменить настройки прибора или перейти непосредственно к измерению. Процесс взаимодействия программы с пользователем однозначно совпадает с возможностями реального прототипа и симулирует на высоком уровне проведение измерений. На рисунке 6 изображено состояние системы во время симуляции измерения оптической плотности бланка, которое характеризуется задержкой на дисплее виртуального прибора сообщения о считывании с помощью таймера.

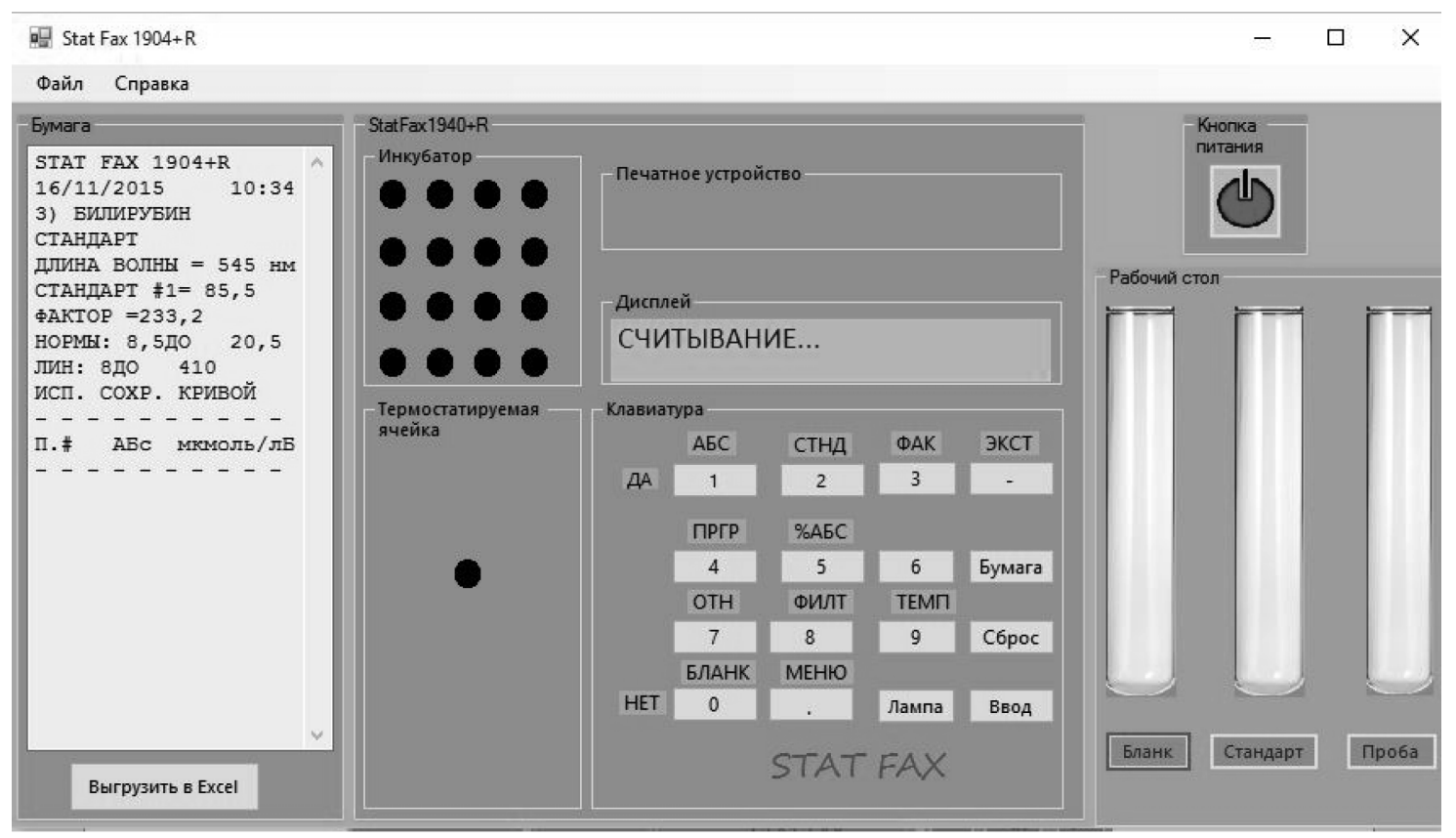

Рис. 6. Виртуальный рабочий стол для проведения биохимического анализа с помощью прибора Stat Fax ${ }^{\circledR} 1904+$. 
Обеспечение достоверности данных было осуществлено посредством защиты системы от некорректного ввода пользователя, позволяющей обучить студента алгоритму работы с виртуальным прибором. Например, если пользователь ошибочно нажимает клавишу, то система выводит предупреждающее сообщение с подсказкой о дальнейшем действии, и после нажатия кнопки «ОК» активными остаются только верные клавиши (рис. 7).

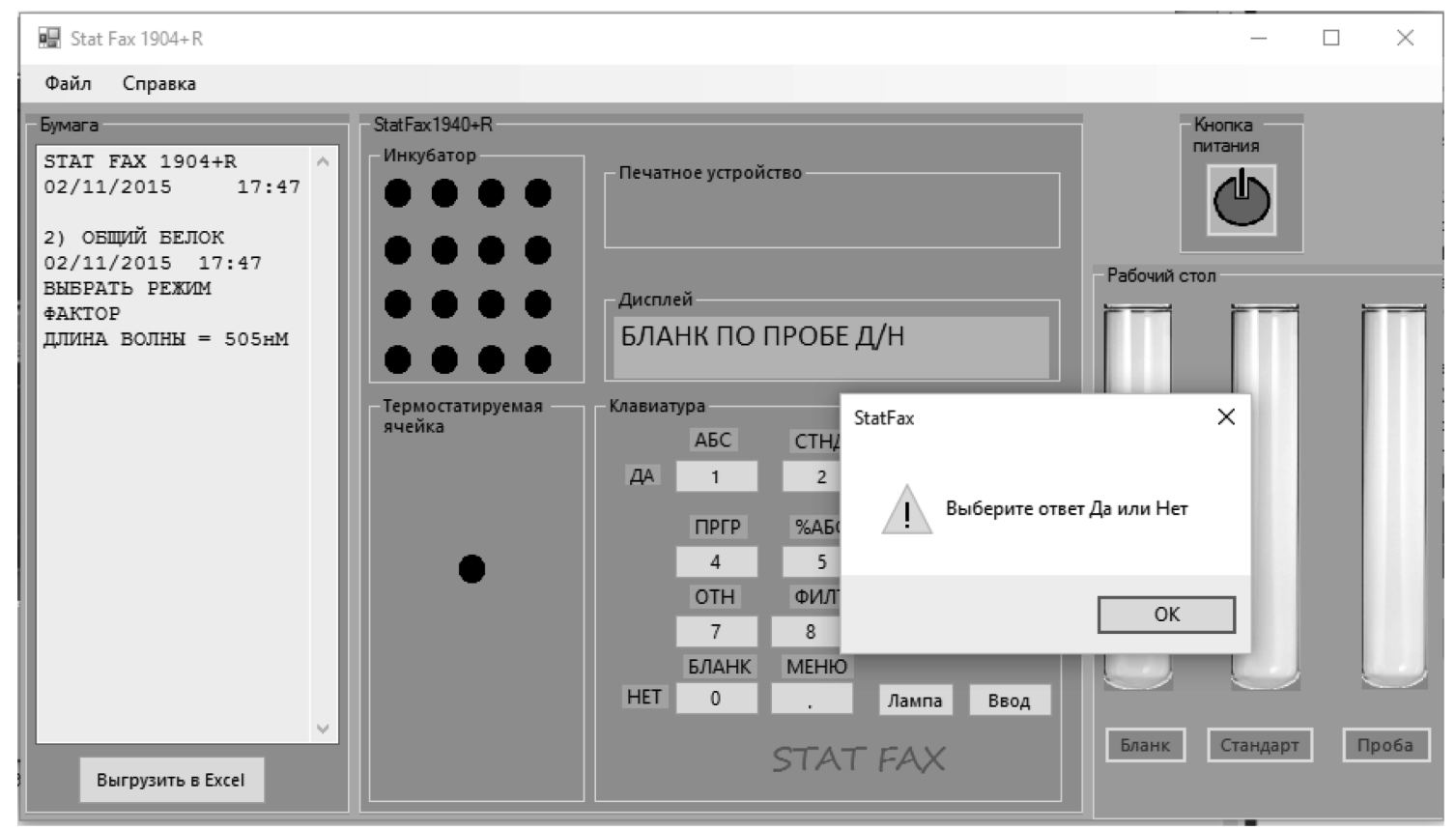

Рисунок 7. Сообщение о некорректном вводе.

\section{Заключение}

Компьютерный симулятор имитирует работу специалиста с прибором Stat Fax ${ }^{\circledR} 1904+$ и позволяет создать виртуальную реальность измерения концентрации биологических жидкостей с помощью биохимического анализатора. Разделение прав пользователей виртуального прибора позволяет организовать индивидуальный подход в образовательном процессе и повысить его эффективность. Разработанная система защиты от некорректного ввода данных способствует отработке алгоритма проведения биохимического анализа. Использование данного симулятора и подобных ему компьютерных программ направлено на сокращение разрыва между современными медицинскими технологиями, применяемыми в клинической лабораторной диагностике, и материально-техническим оснащением образовательного процесса, не снижая при этом качества обучения. Применение симулятора клинического лабораторного анализа будет способствовать не только формированию профессиональных компетенций будущего специалиста, но и предотвращению износа оборудования, экономя финансовые средства. 


\section{Библиография :}

1. Blum T., Rieger A., Navab N., Friess H., Martignoni M. Review of Computer-Based Simulators for Ultrasound Training / T. Blum, A. Rieger, N. Navab, H. Friess, M. Martignoni // Simulation in Healthcare: The Journal of the Society for Simulation in Healthcare. - 2013. - Vol. 8 (2). - P. 98-108.

2. Chung, Chris. Simulation modeling handbook: a practical approach / Chris. Chung. - NY: CRC Press LLC, 2004. - 574 p.

3. Crowe M.E., Hayes Ch.T., Hassan Z.-U. Using Software-Based Simulation for Resident Physician Training in the Management of Temporary Pacemakers / M.E. Crowe, Ch.T. Hayes, Z.-U. Hassan // Simulation in Healthcare: The Journal of the Society for Simulation in Healthcare. - 2013. - Vol. 8 (2). - P. 109-113.

4. Kolsanov, A.V. Virtual surgeon system for simulation in surgical training / A.V. Kolsanov, A.V. Ivaschenko, A.V. Kuzmin, A.S. Cherepanov // Biomedical engineering. - 2014. - Vol. 47 (6). - P. 285-287.

5. Moreno-Ger, P. Application of a low-cost web-based simulation to improve students' practical skills in medical education / P. Moreno-Ger, J. Torrente, J. Bustamante, C. Fernández-Galaz, B. Fernández-Manjón, M.D. Comas-Rengifo // International Journal of Medical Informatics. - 2010. - Vol. 79 (6). - P. 459-467.

6. Долгов В.В., Ованесов Е.Н., Щетникович В.А. Фотометрия в лабораторной практике / В.В. Долгов, Е.Н. Ованесов, В.А. Щетникович. - СПб.: Витал Диагностикс, 2004. - 192 с.

7. Меньшиков В.В. Лабораторный специалист и клиническая интерпретация лабораторных результатов / В.В. Меньшиков // Клиническая лабораторная диагностика. - 2014. - Т. 59 (5). - С. 60-64.

8. Гуляихина Е.С. Компьютерная симуляция проведения биохимического анализа в медицинском образовании // Кибернетика и программирование. - 2014. - 4. - С. 46 - 56. DOI: 10.7256/2306-4196.2014.4.12677.

URL: http://www.e-notabene.ru/kp/article_12677.html

\section{References:}

1. Blum T., Rieger A., Navab N., Friess H., Martignoni M. Review of Computer-Based Simulators for Ultrasound Training / T. Blum, A. Rieger, N. Navab, H. Friess, M. Martignoni // Simulation in Healthcare: The Journal of the Society for Simulation in Healthcare. - 2013. - Vol. 8 (2). - P. 98-108.

2. Chung, Chris. Simulation modeling handbook: a practical approach / Chris. Chung. - NY: CRC Press LLC, 2004. - 574 p.

3. Crowe M.E., Hayes Ch.T., Hassan Z.-U. Using Software-Based Simulation for Resident Physician Training in the Management of Temporary Pacemakers / M.E. Crowe, Ch.T. Hayes, Z.-U. Hassan // Simulation in Healthcare: The Journal of the Society for Simulation in Healthcare. - 2013. - Vol. 8 (2). - P. 109-113.

4. Kolsanov, A.V. Virtual surgeon system for simulation in surgical training / A.V. Kolsanov, A.V. Ivaschenko, A.V. Kuzmin, A.S. Cherepanov // Biomedical engineering. - 2014. - Vol. 47 (6). - P. 285-287.

5. Moreno-Ger, P. Application of a low-cost web-based simulation to improve students' practical skills in medical education / P. Moreno-Ger, J. Torrente, J. Bustamante, C. Fernández-Galaz, B. Fernández-Manjón, M.D. Comas-Rengifo // International Journal of Medical Informatics. - 2010. - Vol. 79 (6). - P. 459-467.

6. Dolgov V.V., Ovanesov E.N., Shchetnikovich V.A. Fotometriya v laboratornoi praktike / V.V. Dolgov, E.N. Ovanesov, V.A. Shchetnikovich. - SPb.: Vital Diagnostiks, 2004. - 192 s. 
7. Men'shikov V.V. Laboratornyi spetsialist i klinicheskaya interpretatsiya laboratornykh rezul'tatov / V.V. Men'shikov // Klinicheskaya laboratornaya diagnostika. - 2014. - T. 59 (5). - S. 60-64.

8. Gulyaikhina E.S. Komp'yuternaya simulyatsiya provedeniya biokhimicheskogo analiza v meditsinskom obrazovanii // Kibernetika i programmirovanie. - 2014. - 4. - C. 46 - 56. D0I: 10.7256/2306-4196.2014.4.12677. URL: http://www.e-notabene.ru/kp/article_12677.html 